\title{
Educación en Época de Covid-19. Una Mirada desde las Diferentes Prácticas Educativas
}

${ }^{1}$ Institución Educativa Técnico San Martin de Tours, Colombia aperfredy@gmail.com

2. Universidad Pedagógica y

Tecnológica de Colombia

Como citar: Aperador, F. E., \& PatinoCuervo, D. (2021). Educación en Época de Covid-19. Una Mirada desde las Diferentes Prácticas Educativas. Educación Y Ciencia, (25), e12624. https://doi.org/10.19053/0120-7105. eyc.2021.25.e12624
Fredy Esteban Aperador ${ }^{1}$

Daniela Patińo-Cuervo ${ }^{2}$

\section{Resumen}

El texto presenta los resultados del proyecto de investigación, el cual pretendió conocer las practicas utilizadas por docentes de una Institución Educativa de la ciudad de Sogamoso, Boyacá, y cómo estas han transformado la realidad de los estudiantes. La perspectiva metodológica se enmarcó en un estudio de corte cualitativo desde un paradigma critico-social. Se utilizó la entrevista a docentes y estudiantes como principal método de recolección de la información. Los resultados del análisis de discursos arrojaron dos grandes vertientes: estrategias didácticas y prácticas pedagógicas, las cuales detallan los procesos educativos que viven tanto docentes como estudiantes en época de pandemia y cómo las estrategias implementadas impactan en el saber de los estudiantes.

Palabras clave: educación, covid-19, practicas pedagógicas, estrategias educativas. 


\title{
Education in Times of Covid-19. A Look from Different Educational Practices
}

\begin{abstract}
The text presents the results of the research project education in times of coronavirus: discourses and practices of students and teachers, which sought to know the practices used by teachers of an educational institution in the city of Sogamoso, Boyacá, and how these have transformed the reality of students. The study used a qualitative methodological perspective from a critical-social paradigm. Interviews with teachers and students were the main method of data collection. The results of the discourse analysis showed two main aspects: didactic strategies and pedagogical practices, which detail the educational processes experienced by both teachers and students in times of pandemic and how the strategies implemented have an impact on the students' knowledge.
\end{abstract}

Keywords: education, covid-19, pedagogical practices, educational strategies.

\section{Introducción}

Este artículo presenta los resultados parciales de la investigación educación en tiempos de coronavirus: discursos y prácticas de estudiantes y docentes, la cual se realizó como trabajo de grado de la Maestría en Educación de la Universidad Pedagógica y Tecnológica de Colombia.

Actualmente, se viven momentos de cambio en los procesos escolares debido a la suspensión de clases presenciales como medida de protección frente la covid-19; distintas escuelas en el mundo tuvieron que tomar medidas apresuradas; tanto docentes como estudiantes se han visto abrumados mientras se define el rumbo adecuado a seguir (Villafuerte, 2020). Según cifras de la Unesco (2020), cerca de 1.370 millones de estudiantes de 138 países se han visto afectados por el cierre parcial o total de jardines, colegios y universidades, lo cual les ha ocasionado estrés por el encierro forzado, histeria colectiva y la creación de entornos virtuales de aprendizaje improvisados por parte de las instituciones educativas. Esto nos lleva a repensar el modo y la forma en que el docente, la escuela y, en general, el sistema educativo debe actuar en tiempos de crisis.

Las adaptaciones, dinámicas y reflexiones escolares realizadas durante pandemia son también una invitación sugerente a maestros e investigadores de la educación para reconsiderar el papel de la escuela en tiempos de crisis y generar espacios académicos que analicen y vinculen diferentes formas de enseñanza e innovaciones curriculares. Así, los aprendizajes derivados de la experiencia, el encuentro y los planteamientos innovadores se configuran como posibilidades para que las instituciones educativas desarrollen procesos de preparación con miras a afrontar las realidades de la sociedad 
actual, las cuales son fruto de diversas situaciones adversas para la humanidad (Oliva, 2020).

De la crisis educativa provocada por la covid-19 han emergido varias opciones metodológicas como plan de contingencia (la educación virtual es una de ellas), lo cual ha implicado cambiar los escenarios presenciales, lo que, a su vez, acarrea transformaciones en el concepto de aula para las prácticas educativas, las estrategias de enseñanza, los hábitos del estudiante y la construcción del conocimiento desde la guía del tutor. No obstante, la virtualidad requiere de ciertas características técnicas y de adaptaciones curriculares para su implementación (Barrios, 2020). He aquí la importancia de recordar que transportar algo (contenidos) del contexto analógico al digital, no implica forzosamente una transformación a modelos virtuales que responda a los intereses formativos de las instituciones educativas (Llorens-Largo, 2020).

Según López (2011), en los procesos escolares, ya sean presenciales o virtuales, se debe buscar la equidad educativa y respetar las diferencias del estudiantado desde la igualdad de oportunidades y el desarrollo de las competencias cognitivas y culturales. Situación que el contexto colombiano no ofrece debido a, entre otros, la baja accesibilidad y conectividad de los hogares, ya que de cada 100 familias, tan solo 13 tienen internet fijo y 57 tienen datos en planes de redes sociales (DANE, 2019). Sumado a ello, el desconocimiento por parte de algunos docentes o de toda la comunidad educativa acerca de las metodologías virtuales, ha implicado, más aún en tiempos de pandemia, improvisación en estrategias, prácticas y dinámicas de aprendizaje que no siempre cumplen sus propósitos educativos o no son asequibles a toda la población (Barrios, 2020).

El problema de conectividad se evidencia en gran medida en las zonas rurales. Durante la pandemia, los niños de estas poblaciones han visto truncado su proceso educativo, pues al no contar con herramientas tecnológicas y al estar la escuela en una dinámica de virtualidad, los procesos escolares han quedado suspendidos o supeditados a lo que los padres de familia puedan enseñar.

Con base en esta problemática surge la pregunta ¿cuáles son las prácticas educativas de estudiantes y docentes de la Institución Educativa Técnico San Martín de Tours de la ciudad de Sogamoso en época de la pandemia de la covid-19? Para dar respuesta a este interrogante, la investigación que da lugar a este artículo se planteó como objetivo principal, analizar las prácticas y discursos de la comunidad objeto de estudio.

\section{Prácticas Pedagógicas}

Para Zaccagnini (2003), las practicas pedagógicas no solo se limitan al ámbito escolar, también se definen como estrategias privilegiadas de las que se vale la sociedad para trasmitir conocimientos y saberes. Pulido (2019) propone que, las practicas 
pedagógicas parten de un adiestramiento que tiene por finalidad la trasmisión de conocimientos para que los individuos puedan integrarse con mayor éxito en la sociedad. En este sentido, todo hecho en el que se trasmitan conocimientos es en sí productor de individuos; a través o gracias a los demás, se construye una persona que se ha de denominar como sujeto pedagógico (Puiggrós, 2002).

Según Paulo Freire (1976) las prácticas educativas en el que hacer pedagógico no deben referirse únicamente a lo que ocurre en la escuela, sino más bien, deben ser consideradas de una manera holística en la que los saberes y conocimientos culturales ofrecidos a las nuevas generaciones por parte de la sociedad, permitan generar posturas críticas, reflexivas y transformadoras que orienten hacia la construcción de una sociedad libre en la que se disminuya la brecha social y no exista distinción de clases.

La práctica pedagógica permite que el docente avance en su quehacer educativo y en la construcción de una reflexión crítica que parta del diario vivir y el discurrir de la sociedad. Tal como afirman Zuluaga y Martínez (1996), la educación como proceso formativo requiere de docentes íntegros y calificados, capaces de interactuar, crear relaciones con los educandos y hacer que los aprendices se enamoren del conocimiento. Esta relación se fundamenta en vínculos comunicativos y asertivos que solo se logran mediante buenas prácticas pedagógicas.

Por ello, la práctica pedagógica debe ser vista dentro de la escuela como una forma de articulación de la producción investigativa, la asimilación de saberes, la experimentación y la apropiación de conocimientos.

\section{Modalidades Educativas en Tiempo de Pandemia}

Durante los confinamientos decretados a causa de la covid-19, los procesos escolares han mutado de modelos basados en la presencialidad a formas que privilegian los medios digitales y la interacción remota, síncrona y asíncrona (Gutierrez-Moreno, 2020). En medio de la crisis, los maestros y la comunidad educativa han diseńado mecanismos de interacción que no responden a la educación virtual, a distancia, en casa o en línea, pero que se fundamentan en ellas y dan como resultado una amalgama de perspectivas y prácticas divergentes. Por ello, es necesario preguntarse ¿en qué consiste cada una de las modalidades educativas que conforman el entramado de interacción y comunicación que se ha dado en la escuela durante la pandemia?

La Educación Virtual [EV] o E-learning es una modalidad de educación a distancia en la que prevalece el uso de internet como principal medio tecnológico (Ruipérez, 2003). La EV es una terminología utilizada principalmente en las universidades para referirse a la educación impartida de manera remota y que, actualmente, se ha trasladado a la educación escolar. El E-learning por su parte, es un término que se usa de manera significativa en el ámbito empresarial y que hace referencia a la capacitación 
remota que se le da a los trabajadores. Sin embargo, es el mismo fenómeno en el que la EV es el resultado de innovaciones en el campo de las Tecnologías de la Información y la Comunicación [TIC], en el cual convergen agentes que, de manera individual, crean nuevos y diversos modos de expandir los procesos de enseńanza-aprendizaje (Ruipérez, 2003).

Es importante aclarar que este tipo de enseñanza no solo debe ser visto desde una perspectiva tecnológica, sino que además debe contemplar la parte humana y social. Para Coll (2008), se debe caracterizar el contexto social actual y dejar claro que vivimos en una dualidad constante, en la que se cuenta con un espacio físico que hace parte de nuestra cotidianidad, pero también se nos brinda la posibilidad de acceder a un nuevo lugar que en realidad es un "no sitio", ya que existe, pero en él no es necesaria la presencialidad. Esto nos lleva a interactuar de forma distinta, a vivir, relacionarnos, comunicarnos diferente y por tanto a aprender de manera diferente" (Angulo, 2016, p.19).

La educación a distancia surge de las innovaciones tecnológicas desarrolladas en el siglo XX e inicios del XXI, las cuales han proporcionado canales de información y comunicación gracias a las redes informáticas y el uso del internet (Facundo, 2003). De acuerdo con lo planteado por Murillo (2017), vivimos en una sociedad que exige una renovación constante del conocimiento y mayor rapidez y fluidez de los procesos educativos. Por ello, los docentes, y en general todo el aparato educativo, deben valerse de la mediación tecnológica para disponer de manera rápida de fuentes de información, a través de diferentes medios que son el resultado del desarrollo acelerado de la tecnología. En este punto, la educación a distancia rompe paradigmas y se proyecta como una estrategia en la que, de forma simultánea, diferida, unidireccional o bidireccional se utilizan tecnologías muy diversas como la televisión, la radio, los videos, los CD, las teleconferencias, las computadoras y el internet. Es así que, en tiempos de aislamiento en los que la educación presencial no es una opción, la educación a distancia toma un papel fundamental en el proceso de formación de los educandos.

El homeschooling o educación en casa es una alternativa educativa que acogen algunos padres de familia para que la enseńanza de sus hijos se de en el hogar (Quiñones, 2018). Esto sin desconocer el sistema educativo tradicional que sustenta su quehacer en la educación obligatoria. Se trata de un modelo de elección cuidadoso en el que los padres, tutores u otras figuras, deciden educar al niño sin vincularlo a las instituciones educativas tradicionales ordenadas por la constitución y la ley. Por ello, esta modalidad no puede considerarse dentro de las situaciones de absentismo escolar, en las que se le niega al menor el derecho fundamental a la educación, más bien, la educación en casa es un esfuerzo suplementario de las familias para garantizar la calidad de la educación de sus hijos, a través de un proceso consciente, organizado y estructurado que adapta los diferentes contenidos educativos a las necesidades y habilidades del menor. 
La educación en casa debe abarcar un modelo de educación básica, en el cual, el niño pueda desarrollarse en el respeto a los principios democráticos de convivencia, los derechos, las libertades fundamentales. Permite articular los contenidos básicos que exige el modelo de educación tradicional para cada nivel, con modelos propios y con pedagogías que concuerden con el modo en que los padres desean que sus hijos sean educados (Soberanes \& Trejo, 2011).

De otra parte, la educación en línea es una estrategia educativa que se encuentra en auge gracias a los avances tecnológicos y en especial a las redes tecnológicas y el internet; es un modelo que empieza a consolidarse a finales del siglo XX con el desarrollo del correo electrónico, los boletines electrónicos y los grupos de discusión virtuales o New Groups. Sin embargo, solo hasta el desarrollo de los navegadores web, a partir de 1993, la educación en línea se afianzó paulatinamente como una posibilidad de enseńanza frente a las ya existentes (Villaverde, 2015). La educación en línea ha ido creciendo horizontalmente gracias al desarrollo aplicado de las TIC (Rama, 2016), lo que también ha permitido la integración de las innovaciones tecnológicas más recientes al catálogo de didácticas e incrementado las opciones de implementación.

Una particularidad de la educación en línea es la opción de asincronía, la cual está relacionada a su origen y evolución, ya que, desde que se configuró como modelo educativo, ha permitido relativizar el tiempo y el espacio a través de la conexión remota. Un estudiante puede acceder al contenido en cualquier momento y lugar, siempre que exista una conexión a internet, lo cual facilita el acceso a la información y permite que la adquisición de conocimientos se haga en diferentes espacios, no solo en la escuela. Esta flexibilidad, favorece la interacción entre los diferentes actores educativos, al no restringir la educación al hecho de la presencialidad y simultaneidad (Salgado, 2015).

La adopción de la educación en línea en época de aislamientos a causa de covid-19, surge como una opción para que los docentes se acerquen más a los estudiantes, sin la necesidad de estar compartiendo el mismo espacio físico. En la actualidad, no es sensato sostener una posición indiferente y apartada de estas nuevas perspectivas de enseñanza; si bien es cierto que su adopción ha causado sentimientos intensos y encontrados de aceptación o rechazo, estos no deben ser impedimento para no implementar o aprobar estos modelos emergentes.

Por otro lado, está la educación remota de emergencia, la cual nace como un nuevo concepto en educación a distancia, producto de trasladar los procesos de aprendizaje que se llevaban de manera presencial hacia la virtualidad. Se remite a perspectivas que no necesariamente se enmarcan dentro de las modalidades a distancia, en casa o en línea (Cabrales et al., 2020). 


\section{Plano Metodológico}

Esta investigación se enmarca dentro de un enfoque cualitativo y descriptivo, que pretende analizar y entender un fenómeno social dentro de un contexto educativo. Para este caso es la educación desarrollada durante el aislamiento por covid-19. El enfoque cualitativo, utiliza la recolección y análisis de datos para afinar las preguntas de investigación y revela nuevos interrogantes en proceso de interpretación (Hernández et al., 2014).

Se abordará este estudio desde un paradigma critico social, ya que tal como afirma Carr y Kemmis (1988):

la ciencia social crítica intenta analizar los procesos sociales e históricos que influyen sobre la formación de nuestras ideas sobre el mundo social (por ejemplo, el papel del lenguaje en el modelado de nuestro pensamiento, o el de los factores económicos o culturales en el modelado de nuestras circunstancias) (p. 368).

En el estudio participaron cinco docentes y cinco estudiantes de la Institución Educativa Técnico San Martín de Tours de la ciudad de Sogamoso. El criterio de selección de los participantes se hizo a conveniencia, tal como mencionan McMillan y Schumacher (2005) "Una muestra por conveniencia es un grupo de sujetos seleccionados sobre la base de ser accesibles o adecuados" (p. 40). Para el caso de los docentes se tuvo en cuenta una antigüedad en la institución superior a dos ańos y para los estudiantes, se eligió a uno por grado desde cuarto hasta grado once.

El instrumento utilizado fue una entrevista estructurada cuyo objetivo fue el de conocer las prácticas y discursos que manejaron los docentes y estudiantes durante el tiempo de aislamiento en época de pandemia covid-19. En esta se plantearon interrogantes tales como: ¿cree usted que este tipo de educación es la adecuada para los estudiantes en época de pandemia? ¿Por qué? ¿Qué entiende por virtualidad?... El trabajo de campo se desarrolló a lo largo del segundo semestre académico del 2020.

Para el análisis de los datos se establecieron categorías y subcategorías que nos permitieron dar cuenta de las practicas y discursos manejados por docentes y estudiantes en época de pandemia, además, estas se fueron ampliando con los elementos emergentes de las entrevistas analizadas, ya que, tal como afirma Rivas (2015) por lo general, "una variable o categoría contienen un factor decisivo en la explicación de un fenómeno" (p. 3). Para la codificación y jerarquización de los datos, se diseńó una matriz en Excel para transformar los datos encontrados en unidades de registro, lo cual permitió profundizar en el estudio de los mismos. Una vez se agruparon y jerarquizaron los códigos, se diseñó la matriz hermenéutica (tabla 1). A cada docente se le asignó la letra $\mathrm{D}$ seguida del número de la entrevista; con los estudiantes se usó la letra E y la numeración consecutiva. 
La tecnología ha permitido incluir nuevas metodologías educativas que inciden en el contexto social e intelectual de las personas; la implementación y articulación de estas se ha convertido en un tema de relevancia tanto en lo pedagógico como tecnológico, ya que repercuten favorablemente en el desarrollo académico de los estudiantes. Proyectos enfocados desde la educación en casa o en línea, se perfilan como nuevas tendencias de gran aceptación, más aún en tiempos de crisis como la generada por la covid-19.

Tabla 1

Codificación de datos

\begin{tabular}{ccc}
\hline \multirow{2}{*}{ Entrevistas } & \multicolumn{2}{c}{ Códigos } \\
\cline { 2 - 3 } & $\begin{array}{c}\text { Estrategias } \\
\text { Didácticas }\end{array}$ & $\begin{array}{c}\text { Practicas } \\
\text { Pedagógicas }\end{array}$ \\
\hline D1 & 8 & 0 \\
\hline D2 & 4 & 2 \\
\hline D3 & 8 & 6 \\
\hline D4 & 0 & 7 \\
\hline D5 & 4 & 9 \\
\hline E1 & 2 & 1 \\
\hline E2 & 1 & 2 \\
\hline E3 & 0 & 3 \\
\hline E4 & 2 & 1 \\
\hline E5 & 1 & 2 \\
\hline
\end{tabular}

Las perspectivas de educación alternativa buscan que la tecnología forme parte del aprendizaje e interacción del estudiantado sin la necesidad de coincidir en un espacio físico. Visto desde otro punto, se trata de generar espacios que no requieran de un tiempo o lugar predeterminado para el proceso educativo.

En el discurso de los participantes fue posible distinguir dos grandes ejes temáticos: estrategias didácticas y prácticas pedagógicas. Los resultados se presentarán acorde a tales bloques.

\section{Resultados}

\section{Estrategias Didácticas}

Para Díaz y Hernández (2002) las estrategias didácticas "son procedimientos que el agente de enseñanza utiliza en forma reflexiva y flexible para promover el logro de aprendizajes significativos en los alumnos" (p. 140). Es decir, involucra medios o recursos mediadores que el docente debe conocer ampliamente, sus funciones, usos y desarrollos en el aula.

La creación de estrategias que potencian el aprendizaje requiere de creatividad, 
interés y reflexión por parte del docente (López, 2017). La nueva realidad educativa causada por la pandemia de la covid-19, ha implicado que los docentes diseñen estrategias didácticas y métodos de enseñanza novedosos para la consecución de logros y metas en el aprendizaje. Así lo comentaron algunos de los docentes entrevistados:

he diseñado guías. Algunas con diseño metodológico, en las cuales dependiendo de la situación que esté cada niño, se modifican para adaptarse a cada una de sus realidades. Además puedo decir que he intentado dosificar los contenidos de tal manera que todos puedan alcanzar la meta [D1].

he utilizado diferentes estrategias, ya sea el trabajo por guías, explicaciones a través de videollamadas, explicaciones a través de llamadas como tal y videos editados por uno mismo para explicar algunos temas [D3].

Así mismo, los programas radiales y televisivos son un medio de instrucción que, combinados con la orientación permanente del docente, permiten una mayor apropiación de las temáticas vistas en clase (Carram et al., 2013). En Colombia, el Ministerio de Educación Nacional $[\mathrm{MEN}]$ ha creado programas de apoyo ${ }^{1}$ que permiten el refuerzo de contenidos y temáticas vistas en el año escolar, sin embargo, estos no son aprovechados debido a falta de acompańamiento suficiente de las familias. Sobre este tipo de estrategias los docentes expresaron que:

Son contenidos interesantes y me gusta la propuesta que tienen en cuanto a la transversalidad, si uno mira el programa, el programa tiene transversalidad y está trabajando en varias áreas. También están enfocados hacia los derechos básicos de aprendizaje... el problema es el mismo, el niño en casa está solo, el niño no está trabajando con el docente orientador al lado [D1].

Son programas en donde se da la participación a los niños, entonces es un punto bastante positivo porque más que el programa como tal, se resaltan también experiencias pedagógicas de los maestros. Además, han mostrado cómo desde casa se puede trabajar con herramientas para aprender un tema afín [D5].

A causa deovid-19, los docentes han tenido que resignificar su labor pedagógica y concepción de la escuela, pues esta última no solo es un espacio físico, sino "un tejido, donde todos construyen su visión del mundo, donde los que recién llegan van mirando aquí y allá, preguntándose, conversando con los otros, con los libros, con los teléfonos, los computadores y todo lo que circunda" (Hirmas \& Cisternas, 2019, p. 10). En este sentido, los docentes han asumido el reto que representan las coyunturas y han diseñado nuevas estrategias didácticas apoyadas en las herramientas

1 El programa 'Profe en Tu Casa' es una estrategia del Ministerio de Educación que tuvo como objetivo responder al reto educativo que supuso el aislamiento preventivo. Se emite por los canales televisivos Señal Colombia y el Canal Institucional; por medio de la Radio Nacional de Colombia y en internet a través de RTVC Play de lunes a viernes. Sus contenidos se centran en temas relevantes y de interés para los niños, en los que en una hora puedan contribuir con el desarrollo de competencias básicas, ciudadanas y socioemocionales. 
tecnológicas con las que se cuenta. Sobre esto un docente afirma que:

es un reto porque hay que resignificar y hay que recrear las estrategias didácticas de la enseñanza, apoyado en todo lo que tiene que ver con las herramientas tecnológicas. Pero más que en las herramientas tecnológicas, la pregunta es ¿cómo se hace para para transmitirlo o hacerle llegar el aprendizaje a los estudiantes? Es ahí cuando hablo de resignificar toda la práctica pedagógica; recrear lo que a estrategia didáctica se refiere [D3].

Una de las herramientas utilizadas por docentes en época de pandemia ha sido el WhatsApp, ya que no consume muchos datos móviles porque reduce la calidad de audio y video al transmitir la información. Además, es un medio que permite la comunicación instantánea entre docentes y estudiantes, lo cual facilita la interacción permanente. En palabras de los docentes entrevistados:

En mi quehacer a diario utilizo únicamente como medio de comunicación el WhatsApp y la llamada telefónica... ¿cómo lo aplico? Trato de hacer la clase un poco más didáctica y por medio de videos muy cortos, porque en el colegio tiene que ser de un minuto la explicación. Trato de explicar la actividad de cada día [D5].

La práctica virtual es conectarme con ellos. Hablar a través de WhatsApp. Por las edades que tienen los de mi grado ya están aprendiendo a manejarlo, son ellos los que están participando, entonces a través de este medio, se está teniendo un contacto directo con el estudiante [D1].

Lo que más he utilizado son los grupos de WhatsApp; debido a las condiciones de la población estudiantil del colegio no es posible realizarlo de otra manera. Son muy pocos los [estudiantes] que tienen acceso a internet para uno establecer clases en tiempo real usando algún tipo de plataforma como Zoom o Meet [D3].

Para los estudiantes, la virtualidad no ha sido un camino fácil, pues al no contar con todas las herramientas tecnológicas, no pueden acceder a los contenidos que los docentes preparan. Si bien los docentes pueden crear muchas estrategias, estas no son asequibles para todos los estudiantes, pues para la aplicabilidad de modelos alternativos a distancia se deben contar con los medios tecnológicos adecuados (Martínez, 2008).

En mi caso solo he podido trabajar con guías y mediante llamadas telefónicas, entonces no he podido entender en muchas ocasiones lo que me envían en las guías y pues me ha tocado en muchas oportunidades dejar en blanco o no hacer el taller [E3].

En sus principales objetivos, la educación virtual se propone aportar a la consecución de logros de aprendizaje por parte de los estudiantes (Fundación 
Universitaria Católica del Norte, 2005), no obstante, durante los aislamientos a causa de la covid-19, este modelo no se ha desarrollado acertadamente debido a problemas de accesibilidad a equipos, recursos y conexión de los estudiantes. Esto ha confluido en que los docentes hayan creado una amalgama de estrategias pedagógicas sin que ello implique desarrollar una práctica virtual como tal. Al respecto afirman que:

Yo pienso que virtual no sería [el modelo educativo]. No. Porque realmente estoy trabajando solamente a través de grupo de WhatsApp, entonces digamos que es un medio de comunicación digital o virtual, pero que se estén haciendo prácticas virtuales no considero que las estemos, o por lo menos en mi caso las esté, utilizando total [D1].

El Ministerio mide a todos los estudiantes de igual manera, cuando tenemos diferencias infinitas. Hay distancias muy grandes con referencia a las zonas geográficas del país. Tenemos estudiantes que inclusive yo creo que ni conocen un computador, entonces no podemos hablar de la enseñanza virtual. Es imposible cuando nuestros papás no tienen los elementos como computadores o celulares adecuados para que los nińos estén conectados [D2].

Con la pandemia y la ausencia de presencialidad, los docentes pasaron de usar el tablero como principal material mediador a utilizar nuevos medios digitales como el WhatsApp y acudir a otros que ya existían como la radio y la televisión; han diseñado estrategias de acuerdo a las realidades y necesidades de los estudiantes, bien sea a través de la creación de guías o mediante contenidos digitales.

\section{Practicas Pedagógicas}

Para Diaz (1990) la práctica pedagógica se refiere a los procedimientos, estrategias y acciones que regulan la interacción, la comunicación, los ejercicios del pensamiento, del habla, de la visión, de las posiciones, oposiciones y disposiciones de los sujetos en la escuela.

La pandemia de la covid-19 irrumpió de manera abrupta en la vida y la escuela, lo cual ha generado que los docentes cuenten con poco espacio para preparar y afrontar la situación, sin embargo, la crisis ha sido una oportunidad para que los educadores aprendan nuevas estrategias y dinamicen sus prácticas y procesos en el aula (Cruz \& Benítez, 2020).

Para mí ha tenido un significado muy marcado, ya que era algo que no esperábamos y nosotros que somos ya mayores nunca habíamos pensado en que esta situación llegará a suceder. Convertir nuestro proceso de enseñanza de lo que estábamos haciendo con todos los medios que disponíamos, a uno digital ... ha sido muy 'especial', porque nos ha servido para aprender como docentes nuevos puntos de vista de enseñanzas a través de la tecnología [D2]. 
La educación remota y mediada por espacios virtuales ha tenido un papel importante en la pandemia y ciertos materiales o recursos como videos cortos y podcast_olvidados en la educación presencial — que ofrecen lecturas propias de la sociedad tecnológica contemporánea, han permitido relacionarse con el engranaje del aprender en medio de la informática y lo tecnológico. De hecho, y de acuerdo con la opinión de un docente participante, estas estrategias pueden llegar a "convertirse en una herramienta para continuar con los procesos de la educación” [D5].

La reflexión sobre el objetivo del quehacer educativo, debe asumirse como un proceso en el que además de la transmisión de conocimientos por parte del profesorado, este debe conocer los estados emocionales de los estudiantes, así como la manera en que ellos aprenden, lo cual aportará a organizar de manera más eficaz y eficiente los proceso de enseñanza-aprendizaje que se implementen, posibilitando un tipo de enseñanza más personalizada, en la que se guía al sujeto desde sus componentes sociales, culturales, emocionales y cognitivos (Thompson \& Aveleyra, 2004).

La educación necesita el contacto físico, el estar ahí, porque la educación no es sólo transmisión de conocimiento, hay un montón de elementos. Está la parte motivacional, la parte de las emociones, el mero contacto con los estudiantes, el estar ahí, el hablarles, el tocarle un hombro al muchacho y decirle hermano ánimo, pilas que usted puede, hágale. Entonces yo creo que no, no creo que la virtualidad sea el futuro la educación, puede ser un soporte o un auxilio, eso sí yo creo [D3].

no solamente es repetir conocimientos. En el aula nosotros estamos trabajando con emociones, estamos formando niños de otra manera, tenemos el contacto físico; me refiero a que tenerlos en el salón de clase hace que el acercamiento pueda ser más profundo [D1].

El escenario de distanciamiento social redujo la interacción física entre el maestro y el estudiante, lo cual ha incidido en el ejercicio pedagógico práctico. El acompañamiento presencial constante se trasladó al contacto intermitente dependiente de las condiciones económicas de los estudiantes, lo que impide el encuentro síncrono para el seguimiento del proceso educativo y el desarrollo efectivo de modelos de educación a distancia o virtuales. Por ello, las prácticas de los docentes se ven restringida por las herramientas tecnológicas y conectividad de los estudiantes (Cardini et al., 2020). Sobre esto un docente manifiesta que:

Para mí hubiera sido [bueno] que los niños tuvieran su tablet o su computador y que al menos tuvieran una hora de internet donde tuviéramos nuestra aula virtual y al menos una hora vernos, hablar con los niños, escucharlos. Así fuera una hora. Pero así este tipo de educación no es la adecuada en esta época, lo ideal era que cada niño contará con su tablet o con su computador y se pudiera conectar [D3]. 
El quehacer docente basa buena parte de su ejercicio de la interacción con los estudiantes (Escobar, 2015), por ello se debe fomentar la continua comunicación para evitar perspectivas que trunquen el proceso de aprendizaje y causen deserción. El modelo alternativo de educación que se ha implementado durante el asilamiento a causa de la covid-19, ha permitido que los padres de familia valoren la labor de los docentes.

nos ha tocado estar en constante comunicación para que los niños no deserten de las instituciones, entonces la labor que cumplimos es muy importante y sin duda los padres de familias también se han dado cuenta que no es fácil ser docente [D5].

Las practicas pedagógicas han sido modificadas a lo largo de la historia según las vivencias y las condiciones humanas (Barragán et al, 2012). Sin embargo, en época de pandemia se han tenido que acelerar esas modificaciones hasta el punto de replantear temas relacionados al quehacer docente, que si bien fue puesto en tela de juicio, se ha demostrado su importancia para la sociedad, la escuela y los sujetos en aspectos como el desarrollo social y emocional (Villafuerte et al., 2020).

\section{Conclusiones}

Esta investigación demuestra la importancia del docente en el aula y en los procesos educativos para mantener, aún en épocas de crisis como la generada por la pandemia, estrategias, recursos y métodos que garanticen la continuidad del proceso de aprendizaje. Si bien es cierto que nadie estaba preparado para las contingencias causadas por la covid-19, gracias a los esfuerzos de los docentes, los estudiantes y los padres de familia, se ha logrado mantener en condiciones aceptables los procesos educativos.

Durante la pandemia las brechas de desigualdad en Colombia se marcaron aún más, lo cual se evidenció en el ámbito educativo en la inaccesibilidad de muchos estudiantes a medios adecuados para recibir y continuar su formación, lo que, a su vez, incidió negativamente en la calidad educativa. Si bien los docentes han diseñado muchas estrategias, estas se limitan a las particularidades de cada estudiante para acceder o contar con medios tecnológicos que permitan su proceso de aprendizaje.

Por otro lado, en estos tiempos de crisis se destaca el desarrollo de diversas estrategias, recursos, métodos e innovaciones educativas por parte de los docentes, que surgen de las necesidades contextuales y tienen en cuenta factores intrínsecos y extrínsecos para una adecuada adquisición de conocimientos. Lo cual, ha permitido el diseño de herramientas totalmente asequibles y cercanas a los estudiantes.

El WhatsApp como herramienta educativa ha sido ampliamente utilizado en pandemia, debido a la facilidad de acceso a los contenidos diseńados; además, al ser un medio de difusión instantánea de texto, permite la comunicación y acercamiento 
entre el docente y el estudiante. Si bien esta aplicación no fue diseñada con fines formativos, se destaca su trascendencia para la continuidad del proceso formativo.

Lo anterior evidencia que la tecnología ha permitido desarrollar nuevas metodologías educativas que inciden en el contexto social e intelectual. La buena implementación y articulación de formas alternativas del aprendizaje se ha convertido en un tema de relevancia, ya que repercute favorablemente en el desarrollo académico de los estudiantes. Proyectos enfocados desde la educación en casa o línea, se perfilan como nuevas tendencias de gran aceptación.

La virtualidad, al menos en el discurso, empieza a ser tomada como una cuestión que atañe directamente a las personas; y es desde este principio que debe ser analizada y enfocada, ya que al perseguir ese fin, es posible entender su desarrollo y acción como un acercamiento casual y espontáneo al proceso educativo, que no irrumpe con las concepciones que se tienen de lo que significa 'educar'. Por ello, se hace necesario construir un campo de estudio que permita sistematizar las investigaciones y reflexiones que brinda la virtualidad en la educación.

El docente como actor fundamental de la educación ha retomado su protagonismo y relevancia durante la pandemia; pese a los modelos y formas alternativas que se han desarrollado durante la contingencia, su labor sirvió para garantizar la continuidad en los procesos formativos, además, fue reconocida y resignificada por los mismos padres de familia.

Finalmente, el estudio mostró la forma en que se ha transformado la escuela para adaptarse a los cambios que exige la sociedad del siglo XXI y sus crisis. Se resalta que la educación y la virtualidad no son polos opuestos que no pueden converger, más bien los vínculos que entre ambas se han creado, las convierten en escenarios que se coadyuvan y vigilan en la tarea de formar ciudadanos.

\section{Referencias}

Angulo, L. (2016). Imaginarios Y Apropiación En TIC: El Caso De Los Docentes De La Institución Educativa Litoral Pacifico De Nuqui [Trabajo de grado, Universidad Pedagógica de Pereira]. https://cutt.ly/ITz0MIi

Barragan, D., Gamboa, A., \& Urbina, J. (2012). Práctica pedagógica (1. ${ }^{a}$ ed.). Ecoe Ediciones.

Barrios, A. (4 de marzo, 2020). COVID-19 abre agenda a la virtualidad de la educación colombiana. Pulzo.com. https://www.pulzo.com/opinion/educacionvirtual-tiempos-coronavirus-PP874848

Cabrales, A., Graham, A., Sahlberg, P., Hodges, C., Moore, S., \& Lockee, B. (2020). Enseñanza Remota de Emergencia. Textos para la discusión (1. ${ }^{a}$ ed.). The learning factory.

Cardini, A., Bergamachi, A., D’Alessandre, V., Torre, E., \& Olliver, A. (2020). 
Educar en Pandemia: entre el aislamiento y la distancia social [Nota técnica]. Banco Interamericano de Desarrollo. http://dx.doi.org/10.18235/0002494

Carr, W., \& Kemmis, S. (1988). Teoría critica de la enseñanza. La investigación-acción en la formación del profesorado. Martínez Roca, S.A.

Carram, M., Soria, G., Llimós, G., \& Gerez, N. (2013). La radio en la escuela ¿Solo un medio para aprender más? Razón y Palabra, (49), 1-9. http://wrww. razonypalabra.org.mx/anteriores/n49/bienal/Mesa\%2013/Ponenciallimos.pdf

Cerdá, A. (2001). Nosotros los maestros, Concepciones de los docentes sobre su quehacer. Universidad Pedagógica Nacional de México

Coll, C. (2008). Psicología de la educación virtual. Morata.

Cruz, O. R., \& Benítez, J. (2020). Las crisis también pueden promover el aprendizaje, impacto del Covid-19 en prácticas docentes. Revista Latinoamericana de Estudios Educativos, 50(Especial), 291-302. https://doi.org/10.48102/rlee.2020.50. especial. 114

Departamento Administrativo Nacional de Estadística [DANE]. (2019). Indicadores básicos de tenencia y uso de Tecnologías de la Información y las Comunicaciones - TIC en hogares y personas de 5 y más años de edad. https://www.dane.gov.co/ files/investigaciones/boletines/tic/bol_tic_hogares_2019.pdf

Díaz, F., \& Hernández, R. (2002). Estrategias Docentes para un Aprendizaje Significativo, Una Interpretación Constructivista. Mc Graw-Hill; Interamericana Editores.

Díaz, M. (1990). De la Práctica pedagógica al texto pedagógico. Pedagogía y Saberes, (1), 14-28. https://doi.org/10.17227/01212494.1pys14.27

Escobar, M. B. (2015). Influencia de la interacción alumno-docente en el proceso enseñanza-aprendizaje. Revista de Tecnología y Sociedad, 5(8). http://www. udgvirtual.udg.mx/paakat/index.php/paakat/article/view/230/347

Facundo, A. (2003). La educación superior a distancialvirtual en Colombia [Reporte]. Iesalc; Unesco. http://unesdoc.unesco.org/images/0013/001399/139922s.pdf

Freire, P. (1976). Educación y cambio. Comisión Ecuménica Latinoamericana de Educación.

Fundación Universitaria Católica del Norte. (2005). Educación virtual. Reflexiones y Experiencias (1. ${ }^{\mathrm{a}}$ ed.). Fundación Universitaria Católica del Norte.

Gutierrez-Moreno, A. (2020). Educación en tiempos de crisis sanitaria: pandemia y educación. Praxis, 16(1), 7-10. https://doi.org/10.21676/23897856.3040

Hernández, R., Fernández, C., \& Baptista, P. (2014). Metodología de la investigación (6a ed.). McGrwall Hill Education 
Hirmas, C., \& Cisternas, T. (2019). Resignificando la escuela en el contexto de pandemia. (1. ${ }^{\mathrm{a}}$ ed.). OEI. https://cutt.ly/ARCh16E

Llorens-Largo, F. (2020). Transformación digital versus digitalización. Universidad, SÍ. https://bit.ly/2tmYFMr

López, H. (2011). Los cinco axiomas de la proyección pedagógica diseńable. Revista Teoría y Didáctica de las Ciencias Sociales, (17), 9-26. https://www.redalyc.org/ pdf/652/65221619002.pdf

Martínez, C. (2008). La educación a distancia: sus características y necesidad en la educación actual. Educación, 17(33), 7-27. https://revistas.pucp.edu.pe/index. $\mathrm{php/educacion/article/view/1532}$

McMillan, J., \& Schumacher, S. (2005). Investigación Educativa (5ª ed.). Pearson Education.

Murillo, W. (2017). Un modelo pedagógico de educación universitaria a distancia para estudiantes en prisión: perspectivas y desafíos. Posgrado y Sociedad. Revista Electrónica del Sistema de Estudios de Posgrado, 15(2), 49-64. https://doi. org/10.22458/rpys.v15i2.1964

Oliva, H. (2020). La Educación en tiempos de pandemias: visión desde la gestión de la educación superior. https://doi.org/10.13140/RG.2.2.27595.54568

Puiggrós, A. (2002). Qué pasó en la educación argentina. Galerna.

Pulido, O. (2019). Sobre el maestro, el entrenamiento, el cuidado de sí y el decir veraz. Praxis \& Saber, 10(22), 9-18. https://doi.org/10.19053/22160159.v10. n22.2019.9303

Quiñones, J. (2018). Del concepto de la "educación en casa» a la «educación en familia» en Colombia. Anuario digital de investigación educativa, 1(1), 307-315. https://doi.org/10.13140/RG.2.2.11337.95845

Rama, C. (2016). La fase actual de expansión de la educación en línea o virtual en América Latina. Universidades, 70, 27-39. https:/www.redalyc.org/ pdf/373/37348529004.pdf

Rivas, L. (2015). La definición de variables o categorías de análisis. En Rivas, L. (Ed), ¿Cómo hacer una tesis? (pp. 107-118). Ediciones Taller Abierto.

Ruipérez, G. (2003). Educación virtual y eLearning. Fundación Auna.

Salgado, E. (2015). La enseñanza y el aprendizaje en modalidad virtual desde la experiencia de estudiantes y profesores de posgrado [Tesis doctoral, Universidad Católica de Costa Rica]. https://cutt.ly/tTz3kjV

Soberanes, J. M., \& Trejo, L. A. (2011). Educación escolarizada vs. educación en casa. Reflexiones sobre la sentencia homeschooling del Tribunal Constitucional 
español. Cuestiones constitucionales, (25), 353-365. http://dx.doi.org/10.22201/ iij.24484881e.2011.25.5977

Thompson, S., \& Aveleyra, E. (2004). Estilos de aprendizaje en Matemática. Memorias de las XIX Jornadas Nacionales de Docentes de Matemática de Facultades de Ciencias Económicas y Afines, Argentina.

Unesco. (24 de marzo, 2020). 1.370 millones de estudiantes ya están en casa con el cierre de las escuelas de COVID-19, los ministros amplian los enfoques multimedia para asegurar la continuidad del aprendizaje. https://es.unesco.org/news/1370millones-estudiantes-ya-estan-casa-cierre-escuelas-covid-19-ministros-amplianenfoques

Villafuerte, J., Bello, J., Pantaleón, Y., \& Bermello, J. (2020). Rol de los docentes ante la crisis del Covid-19, una mirada desde el enfoque humano. REFCalE, 8(1), 134158. http://www.refcale.uleam.edu.ec/index.php/refcale/article/view/3214/1986

Villafuerte, P. (4 de mayo, 2020). El aprendizaje remoto enfrenta otro reto: el profesorado no está preparado para la enseñanza en línea. https://www.magisterio. com.co/articulo/el-aprendizaje-remoto-enfrenta-otro-reto-el-profesorado-noesta-preparado-para-la-ensenanza

Villaverde, M. (2015). La educación a distancia y su relación con las nuevas tecnologías de la información y las comunicaciones. Medisur, 11(3), 280-295. http://scielo.sld.cu/pdf/ms/v11n3/ms06311.pdf

Zaccagnini, M. (2003). Impacto de los paradigmas pedagógicos históricos en las prácticas educativas contemporáneas. Revista Iberoamericana de Educación, 33(2), 1-29. https://doi.org/10.35362/rie3322982

Zambrano, A. (2016). Pedagogía y didáctica: esbozo de las diferencias, tensiones y relaciones de dos campos. Praxis \& Saber, 7(13), 45-61. https://doi. org/10.19053/22160159.4159

Zuluaga, O., \& Martínez, A. (1996). Historia de la educación y la pedagogía: desplazamientos y planteamientos. En A. Martínez \& M. Narodowski (Comps.), Escuela, historia y poder: miradas desde América Latina (pp. 55-75). Novedades Educativas. 